Annuaire du Collège de France 2017-2018

\title{
Évolution du climat et de l'océan
}

\section{Édouard Bard}

\section{OpenEdition}

\section{Journals}

Édition électronique

URL : https://journals.openedition.org/annuaire-cdf/15716

DOI : 10.4000/annuaire-cdf.15716

ISBN : 978-2-7226-0572-5

ISSN : 2109-9227

Éditeur

Collège de France

Édition imprimée

Date de publication : 30 décembre 2020

Pagination : 109-120

ISBN : 978-2-7226-0516-9

ISSN : 0069-5580

Référence électronique

Édouard Bard, «Évolution du climat et de l'océan », L'annuaire du Collège de France [En ligne], 118| 2020, mis en ligne le 01 avril 2021, consulté le 22 août 2022. URL : http://journals.openedition.org/ annuaire-cdf/15716 ; DOI : https://doi.org/10.4000/annuaire-cdf.15716 


\title{
ÉVOLUTION DU CLIMAT ET DE L'OCÉAN
}

\author{
Édouard BARD \\ Membre de l'Institut (Académie des sciences), \\ professeur au Collège de France
}

Mots-clés : climat, océan, niveau marin

Le colloque "Le climat des deux derniers millénaires " est disponible en vidéo sur le site internet du Collège de France (https://www.college-de-france.fr/site/edouardbard/symposium-2017-2018.htm).

\section{ENSEIGNEMENT}

COURS - ClimATS EXTRÊMES ET ANALOGUES ACTUELS : PETIT ÂGE GLACIAIRE ET OPTIMUM CLIMATIQUE MÉDIÉVAL

Il est impératif de remettre le réchauffement du dernier siècle dans un contexte temporel plus large afin d'en déterminer la singularité et de distinguer les causes sous-jacentes, naturelles et anthropiques. Les variations climatiques ne se répètent jamais à l'identique, mais les mêmes mécanismes physiques, chimiques et biologiques sont à l'œuvre et peuvent faire l'objet de simulations numériques avec les mêmes modèles utilisés pour prévoir les futurs possibles. Le climat du dernier millénaire peut donc servir à tester de multiples hypothèses avec une fiabilité des enregistrements et une couverture spatiale généralement suffisantes. L'étude de cette période permet en outre de considérer l'impact du climat sur les sociétés humaines et d'en étudier les réactions. Ces leçons de l'histoire peuvent nous éclairer dans nos choix actuels, même si notre société mondialisée est évidemment bien différente en raison de sa démographie et de sa connectivité à l'échelle planétaire.

\section{Cours 1 - Températures du dernier millénaire à l'échelle globale}

Le climat a évolué au cours du dernier millénaire. C'est un fait reconnu depuis les travaux pionniers du météorologue anglais Hubert Lamb, fondateur du Climate Research Unit de l'université d'East-Anglia, et de l'historien français Emmanuel Le 
Roy Ladurie, titulaire de la chaire Histoire de la civilisation moderne au Collège de France (1973-1999). Des mesures thermométriques directes ont permis de reconstituer des séries remontant jusqu'au XVII ${ }^{\mathrm{e}}$ siècle, notamment en Angleterre et aux Pays-Bas. Ces séries présentent néanmoins de multiples problèmes qui doivent être corrigés avant toute interprétation, comme l'illustre la série pluriséculaire de la température de la ville de Bologne.

Pour remonter avant l'invention du thermomètre, il est possible d'exploiter des données climatiques indirectes, comme la perception qualitative de la température, du vent, de la pluie et des nuages, ainsi que l'observation d'événements extrêmes comme les tempêtes, le niveau des rivières et leurs crues, la durée saisonnière du gel et de la couverture de neige. En plus de ces indications d'ordre météorologique, il est aussi utile de se tourner vers d'autres indicateurs, comme les cycles saisonniers des végétaux et des animaux, les types de culture ainsi que les dates et rendements des récoltes.

La première courbe climatique du millénaire publiée par Lamb était centrée sur l'Angleterre. Fondée sur des températures instrumentales et des indicateurs indirects, elle a permis d'introduire les notions d'optimum climatique médiéval (OCM) et de petit âge glaciaire (PAG).

Ce type de reconstitution a été généralisé à la Terre entière, en ajoutant notamment des séries dendroclimatiques fondées sur la relation entre le climat et l'épaisseur ou la densité des cernes d'arbres. Cette relation est partielle et complexe et n'a souvent qu'une validité locale. Les spécialistes privilégient l'étude d'arbres isolés dans des conditions de stress climatique, en limite de zone de dominance géographique ou altitudinale. D'autres archives paléoclimatiques avec des résolutions temporelles variables (mensuelle à décennale) permettent de compléter les séries dendroclimatiques. Il s'agit de séries mesurées dans des sédiments lacustres, des glaces des calottes polaires et des glaciers de montagnes ou bien encore de stalagmites prélevées en grottes.

Le programme international PAGES2k dédié à la compilation d'environ un millier de ces séries a conduit au calcul de courbes moyennes à l'échelle mondiale ou régionale pour les deux derniers millénaires. La courbe d'anomalies de température présente un lent refroidissement $\left(<0,5^{\circ} \mathrm{C}\right)$ depuis l'optimum climatique médiéval jusqu'au petit âge glaciaire, suivi d'une remontée rapide depuis le XIX $\mathrm{X}^{\mathrm{e}}$ siècle. La comparaison des courbes moyennes des deux hémisphères montre quelques découplages d'amplitude et de durée limitées $\left(<0,2{ }^{\circ} \mathrm{C}\right.$ pendant un siècle).

L'étude statistique des moyennes régionales montre que la transition entre la fin du PAG et le réchauffement récent débuterait dès la première moitié du XIX ${ }^{\mathrm{e}}$ siècle. C'est notamment le cas des moyennes pour l'Europe et l'Arctique. Une vue complémentaire est fournie par la détection statistique de l'émergence du réchauffement par rapport à la variabilité naturelle pendant le PAG. Cette sortie du «bruit naturel » intervient au $\mathrm{XX}^{\mathrm{e}}$ siècle : précoce et marquée en zone arctique, plus tardive en Europe, Asie et dans la zone intertropicale, encore difficile à distinguer aux latitudes les plus hautes de l'hémisphère austral.

\section{Cours 2 - Températures de l'océan, variations spatiales et modélisation}

Les mesures instrumentales de la température de surface des océans montrent que le réchauffement au cours $\mathrm{du} \mathrm{XX}^{\mathrm{e}}$ siècle est globalement parallèle à celui de l'atmosphère. Quelques séries remontent jusqu'au XIX ${ }^{\mathrm{e}}$ siècle pour certains sites portuaires. Les mesures de température des premières expéditions scientifiques, par exemple du navire HMS Challenger de 1872 à 1876, peuvent être comparées aux 
données récentes du programme Argo. Pour couvrir les derniers siècles, il est aussi nécessaire de faire appel à des indicateurs paléothermométriques mesurés dans des archives marines (sédiments, coraux, mollusques, etc.) ayant des résolutions temporelles variables (mensuelle à décennale).

Des dizaines de séries réparties dans les trois océans principaux ont été comparées et compilées dans le cadre du programme PAGES2k. Les moyennes montrent que l'océan de surface s'est refroidi au cours de la période historique de façon parallèle à l'atmosphère pour atteindre un minimum vers le début du XIX ${ }^{\mathrm{e}}$ siècle avant l'inversion de tendance marquée par le réchauffement mondial. En plus des données paléocéanographiques directes, il est aussi possible d'utiliser les séries continentales dominées par les données dendroclimatiques. L'étude des covariances spatiales océancontinent pour le dernier siècle permet d'extrapoler la couverture spatiale des variations océaniques pour les périodes plus anciennes. Ceci conduit à la cartographie d'anomalies de température pour les périodes du PAG et de l'OCM. La différence de ces anomalies confirme que la période de l'OCM était globalement plus chaude que celle du PAG, notamment sur les continents (Europe et Amérique du Nord) et les océans, en particulier l'Atlantique et le Pacifique Nord. La carte des différences entre l'OCM et le PAG suggèrent la présence d'une anomalie négative (i.e. OCM plus froid que le PAG) dans la zone équatoriale du Pacifique qui serait liée à une modulation du phénomène ENSO pendant la transition OCM-PAG. Néanmoins, les séries paléocéanographiques réalisées à partir d'archives à résolution saisonnière comme les coraux massifs ne confirment pas de variation marquée du phénomène ENSO pendant le PAG.

Les modèles numériques du climat sont utilisés pour mieux comprendre les changements de température du dernier millénaire, notamment la transition de l'OCM vers le PAG. Il est nécessaire de tenir compte des forçages externes pour cette période: variations orbitales de l'insolation, de l'éclairement solaire, des éruptions volcaniques majeures (i.e. avec injection de $\mathrm{SO}_{2}$ dans la stratosphère), et des teneurs en gaz à effet de serre $\left(\mathrm{CO}_{2}\right.$ et $\left.\mathrm{CH}_{4}\right)$. Ces trois derniers forçages sont notamment reconstitués à partir de mesures réalisées sur les glaces polaires (cosmonucléides, sulfate et anomalie isotopique $\Delta^{33} \mathrm{~S}$, pression partielle dans les bulles d'air occluses dans les glaces).

La comparaison des simulations numériques avec les anomalies de température observées pour le dernier millénaire montre une correspondance satisfaisante pour les tendances et les amplitudes. Le programme international CMIP5 a permis la comparaison et la compilation des simulations climatiques par une dizaine de modèles. Chaque modèle permet de réaliser des simulations séparant les différents forçages climatiques pour en étudier les impacts relatifs. L'étude fine, aux échelles globale et régionales, de l'impact climatique d'une douzaine de grandes éruptions volcaniques depuis $1400 \mathrm{AD}$ démontre la fiabilité des modèles climatiques pour simuler ces refroidissements complexes dont l'amplitude est d'environ $0,3{ }^{\circ} \mathrm{C}$. Les moyennes des simulations pour les grandes zones géographiques peuvent être comparées aux séries observées pour les mêmes régions. La compilation des simulations permet de dater la fin du PAG et le début du réchauffement global à la première moitié du XIX ${ }^{\mathrm{e}}$ siècle. Les simulations suggèrent que la transition est détectable à toutes les latitudes alors que les observations ne sont pas aussi claires pour les hautes latitudes de l'hémisphère Sud. Les simulations moyennes permettent d'établir une cartographie des différences de température entre l'OCM et le PAG. La carte globale montre un réchauffement OCM-PAG beaucoup plus uniforme à l'échelle de la planète sans refroidissement équatorial au niveau de l'océan Pacifique. 


\section{Cours 3 - Niveau marin du dernier millénaire et glaces continentales}

L'évolution du niveau marin est suivie actuellement grâce à un réseau de marégraphes installés sur les côtes ainsi que par les satellites altimétriques depuis les années 1990. La couverture spatiale des marégraphes est assez faible au début du $\mathrm{XX}^{\mathrm{e}}$ siècle et seules quelques stations portuaires permettent de remonter au $\mathrm{XIX}^{\mathrm{e}}$ siècle. Par ailleurs, le niveau observé localement est affecté par une composante géophysique mise en évidence dès le XVIII ${ }^{\mathrm{e}}$ siècle par Anders Celsius. Ce réajustement isostatique postglaciaire se poursuit encore aujourd'hui comme le montrent les mesures actuelles par GPS ainsi que la modélisation physique du phénomène. La correction de cette composante géophysique entraine des biais et des incertitudes, comme on peut l'illustrer avec l'enregistrement moyen du niveau marin en mer Baltique. Au niveau mondial, le niveau moyen montre une augmentation régulière d'environ $25 \mathrm{~cm}$ depuis le milieu du XIX ${ }^{\mathrm{e}}$ siècle.

Pour remonter au-delà, il est nécessaire de faire appel à des indicateurs biologiques comme les micro-atolls en zone intertropicale, les trottoirs algaires sur les côtes rocheuses ou les fossiles de flore et faune adaptées aux gradients de salinité des zones côtières (par exemple, marais salés, salt marsh en anglais). Plusieurs exemples de séries du niveau marin sont présentés pour les côtes provençale et bretonnes, les atolls du Pacifique, la côte est des États-Unis et le Groenland. Comme pour les enregistrements actuels, il est crucial de corriger les séries millénaires des effets modélisés du réajustement postglaciaire qui souvent dominent l'amplitude des variations observées (par ex. sur la côte nord-américaine ou les atolls du Pacifique).

La courbe résiduelle des anomalies du niveau marin au cours du dernier millénaire présente des fluctuations d'amplitude faible d'environ $10 \mathrm{~cm}$ avec un maximum des années 400 à 1000 suivi par un minimum de 1200 à 1850 . Le détail des causes des variations du niveau marin est disponible pour la période couverte par les mesures des satellites. L'augmentation actuelle de $3 \mathrm{~mm} / \mathrm{an}$ est liée pour un tiers à la dilatation thermique de l'océan et pour deux tiers à des flux d'eau douce provenant des continents, notamment les calottes antarctique et groenlandaise et les glaciers de montagne. Les deux composantes sont liées in fine au réchauffement mondial qui se propage dans l'océan et entraîne la fonte des glaces continentales. Les modèles climatiques couplés ont encore des difficultés pour simuler ces deux composantes au cours du dernier millénaire, mais il est possible d'utiliser des relations statistiques calibrées sur le dernier siècle. Les anomalies de température observées pour les deux derniers millénaires peuvent être converties en termes de niveau marin, conduisant à des variations d'environ $10 \mathrm{~cm}$ avec un maximum correspondant à l'OCM et un minimum pour le PAG, en accord avec les observations du niveau marin corrigés des effets géophysiques.

\section{Cours 4 - Le climat du dernier millénaire en Europe et en Arctique}

Les séries d'observations et les modélisations peuvent être restreintes géographiquement à l'Europe. La comparaison modèles-données au cours du dernier millénaire montre encore une correspondance avec les périodes de l'OCM et du PAG, auxquelles se superposent des variations séculaires d'environ $0,5^{\circ} \mathrm{C}$ en amplitude. Les conséquences sont particulièrement visibles dans les Alpes où l'on observe une cohérence des avancées et retraits des glaciers, ainsi que des épisodes de sédimentation rapide dans les lacs de montagne. Les enregistrements à haute 
résolution obtenus pour les Alpes et les zones côtières allant du Maroc à la Scandinavie montrent des variations rapides attribuées à une modulation de l'oscillation nord-atlantique (NAO). Cette oscillation atmosphérique conduit à la variation de l'intensité et de la localisation des dépressions traversant l'Atlantique d'ouest en est. Des séries mesurées sur les glaces du Groenland, des stalagmites et d'arbres en des sites variés, ont permis de construire un indice NAO paléoclimatique pour le dernier millénaire. Ces recherches ont conduit à proposer l'hypothèse d'une oscillation NAO bloquée en phase positive pendant l'OCM, mais les compilations et modélisations récentes montrent des effets assez faibles en amplitude.

Les variations climatiques en Atlantique Nord jusqu'en zone arctique sont étudiées à partir de sédiments océaniques et d'organismes marins présentant des squelettes calcaires avec des cernes annuels (praire d'Islande, algues rouges encroûtantes). La compilation des séries marines montre la succession classique de l'OCM et du PAG, avec un impact sur l'extension de la banquise.

Ces fluctuations climatiques et hydrologiques ont certainement pu favoriser les voyages maritimes des vikings durant l'OCM, incluant la colonisation de l'Islande et du Groenland et l'exploration de la côte de l'Amérique du Nord. La détérioration climatique du PAG a clairement impacté les colonies vikings du Groenland qui se sont adaptées à leur environnement, contrairement à certaines idées reçues. Cet exemple emblématique permet de montrer la complexité des influences sur ces sociétés humaines qui ne se résument pas à l'impact direct de la température. On peut citer les relations conflictuelles avec les Inuits et la chute du commerce de l'ivoire de morse alliée à la baisse des contacts avec l'Europe ravagée par la peste noire. Des travaux récents montrent d'ailleurs que la contamination par la peste est modulée par le climat en Asie centrale qui affecte les grandes gerboises et les puces, vecteurs de la bactérie. Les variations climatiques pendant le PAG semblent même être à l'origine des réintroductions multiples depuis la peste noire.

\section{Cours 5 - Le climat du dernier millénaire en Europe et en Arctique}

Les comparaisons modèles-données pour le dernier millénaire sont en accord pour la longue tendance OCM-PAG, ainsi que pour des refroidissements brefs correspondant à des éruptions volcaniques majeures, parfois rapprochées dans le temps (par exemple 1230-1258, 1809-1815 AD) auxquelles se surimposent les variations de l'activité solaire. La transition OCM-PAG est bornée par les deux éruptions stratosphériques des volcans Samalas en 1257 et Kuwae en 1458 AD. Le flux de $\mathrm{SO}_{2}$ de l'éruption de 1257 est à l'origine de la plus grande perturbation radiative au cours de la période historique. Les stigmates directs sont encore bien visibles sur l'île de Lombok qui a été complètement recouverte de cendres volcaniques. L'impact climatique a été démontré jusqu'en Europe, notamment pour l'année qui a suivi l'éruption avec une diminution des températures, des précipitations anormales et des inondations, qui ont conduit à des baisses des récoltes, une augmentation du prix du grain, accompagnée parfois de famine. Le même scénario a dû se reproduire pour les autres grandes éruptions, notamment celle de 1458 qui débute la période du PAG.

Les données les plus précises concernent l'une des dernières éruptions majeures du PAG : en 1815, les coulées pyroclastiques et les cendres du volcan Tambora ont entraîné la mort de plus de 10000 personnes sur l'île de Sumbawa. Cette éruption a aussi engendré une perturbation radiative mondiale à l'origine de la célèbre « année 
sans été », bien documentée dans les archives météorologiques de 1816 avec une baisse marquée des températures et une augmentation des précipitations en Europe de l'Ouest.

Comme le montrent les données météorologiques et paléoclimatiques pour les périodes entourant ces grandes éruptions ainsi que pour la dernière de ce type d'une ampleur plus faible (mont Pinatubo en 1991), l'impact ne se limite pas au refroidissement par augmentation de la réflexion du rayonnement solaire incident par les aérosols volcaniques. Des distributions complexes de la température sont mises en évidence en hiver, ainsi que des modifications de la dynamique atmosphérique liées à des effets contrastés suivant l'altitude et les latitudes. Les éruptions «stratosphériques » semblent perturber les oscillations nord-atlantique (NAO) et arctique (AO).

La modélisation numérique de la dispersion des aérosols volcaniques et des impacts sur climat permet de tester les hypothèses et de comparer les simulations avec les séries observées pour les températures et les précipitations. Un résultat robuste est une baisse marquée des pluies de la mousson asiatique. À la suite de l'éruption de 1815 du Tambora, la perturbation climatique a entraîné une famine liée à de mauvaises récoltes, accompagnée d'une épidémie de choléra en baie du Bengale. La globalisation des échanges au XIX ${ }^{\mathrm{e}}$ siècle a conduit à une diffusion de la pandémie en Europe puis en Amérique du Nord. L'impact climatique du Tambora s'est aussi fait ressentir en Europe et aux États-Unis avec une flambée du prix du grain. Des études statistiques focalisées sur la démographie de l'Allemagne montrent des correspondances entre les fluctuations météorologiques, le prix du grain et l'émigration des populations vers l'Amérique. À la fin du XIXe siècle, le climatologue Eduard Brückner fut l'un des premiers à étudier l'influence directe du climat sur les récoltes et les conséquences sur ses concitoyens.

Même s'il ne s'agit pas d'une éruption «stratosphérique » classique, l'éruption fissurale du volcan Laki en Islande (1783-1784) a injecté suffisamment de $\mathrm{SO}_{2}$ dans la haute atmosphère pour avoir un impact climatique à l'échelle continentale. Des effets inhabituels seraient liés à la conjonction des émissions volcaniques et de situations météorologiques particulières responsables de la propagation dans toute l'Europe du «brouillard» du Laki. Les impacts sur la température et les précipitations sont bien documentés, notamment en France (par exemple, Seine gelée et crues dans le nord). La perturbation des récoltes qui a suivi l'année 1784 a certainement eu un rôle dans les révoltes qui ont précédé la Révolution française, mais il est difficile de distinguer le rôle du climat par rapport aux déterminants sociologiques de cet événement politique majeur et singulier.

Pour aller au-delà de l'étude d'un événement unique, des chercheurs ont étudié les correspondances statistiques entre les séries climatiques et le nombre annuel de conflits guerriers en Europe. Une corrélation négative entre la température et les guerres semble robuste pour le PAG, période pendant laquelle l'Europe préindustrielle était encore tributaire de son agriculture.

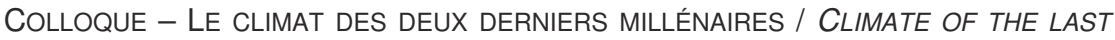
TWO MILLENNIA

Le climat a varié au cours des deux derniers millénaires; son étude permet de replacer le réchauffement des dernières décennies dans le contexte plus large des variations d'origines naturelles. S'agissant de la période historique, il est aussi possible d'évaluer les impacts complexes et multiples sur les sociétés humaines. 
Après avoir exposé le contexte général, le professeur Édouard Bard a illustré la complémentarité des séries thermométriques instrumentales et des indicateurs paléoclimatiques pour reconstituer des anomalies de température à l'échelle des grandes régions de la planète sur les deux derniers millénaires. Il a aussi introduit l'approche par simulation numérique utilisant des modèles de circulation générale perturbés par les variations des forçages externes (gaz à effet de serre, émissions volcaniques, éclairement et insolation).

L'apport fondamental de l'étude des cernes d'arbres a été décrit par Jan Esper, professeur à l'université de Mayence, qui a insisté sur les problèmes de calibration des signaux dendroclimatiques et sur la nécessité de la réplication des séries à partir d'arbres provenant d'une même région. Il a aussi distingué l'intérêt des variations de hautes fréquences par rapport aux tendances à long terme des séries dendroclimatiques.

La complexité de la réponse des sociétés humaines a été abordée par Elena Xoplaki, chercheuse à l'université de Giessen, qui a détaillé le cas du monde byzantin pendant la période correspondant à l'optimum climatique médiéval et le petit âge glaciaire. La résilience et la vulnérabilité de cette société fondée sur l'agriculture sont étudiées afin de discerner l'influence du climat sur les événements historiques de cette région (période des croisades, du sultanat mamelouk, de la conquête ottomane, et des révoltes des Celali...).

Les civilisations humaines installées sous les tropiques sont souvent sensibles aux aléas climatiques. Gerald Haug, directeur à l'institut Max-Plank de chimie de Mayence et professeur à l'ETH de Zürich, a montré l'utilité des séries paléoclimatiques pour étudier les changements de la pluviosité et de la fréquence des sécheresses afin de mieux comprendre les phases d'expansion et de régression des sociétés anciennes de la Mésoamérique et de l'Asie du Sud-Est.

Le professeur Jürg Luterbacher de l'université de Giessen a poursuivi en considérant les variations de température et de précipitation de la zone indochinoise. Les avantages et inconvénients des séries dendroclimatiques ont été abordés pour les périodes correspondant à l'optimum médiéval et au petit âge glaciaire.

L'approche par modélisation numérique a été décrite par Myriam Khodri, chercheuse IRD au LOCEAN-IPSL de Paris, qui a insisté sur la complexité des variations climatiques liées à la combinaison de fluctuations intrinsèques du système climatique avec les variations forcées par les causes externes au système.

Poursuivant sur l'utilisation de la modélisation climatique, Johann Jungclaus, chercheur à l'institut Max-Planck de météorologie de Hambourg, a montré le rôle de la dynamique atmosphérique et océanographique en Atlantique Nord pour expliquer les fluctuations de température et de précipitations observées au niveau régional en Europe et en Eurasie au cours du dernier millénaire.

Le colloque a permis d'illustrer l'importance et la diversité des recherches sur les variations climatiques de la période historique. Les débats nous ont aussi permis d'identifier les incertitudes et les problèmes nécessitant de nouveaux travaux scientifiques pour progresser dans la compréhension des climats passés et dans la prévision des variations futures d'origines humaines et naturelles.

- Édouard Bard (Collège de France \& CEREGE, Aix-en-Provence) : «Introduction and perspective on climate forcings and proxies »;

- Jan Esper (Johannes Gutenberg University, Mainz) : «Millennial-length largescale temperature reconstructions from tree-rings: knowns and unknowns »; 
- Elena Xoplaki (Justus Liebig University, Giessen) : «Climate impacts and societal resilience in the Mediterranean of the last millennium; the case of medieval Byzantium »;

- Gerald Haug (Max Planck Institute for Chemistry, Mainz and Department of Earth Sciences, ETH Zürich): "The role of tropical climate dynamics on the decline of civilizations »;

- Jürg Luterbacher (Justus Liebig University, Giessen) : «New evidence on Asian temperature and hydroclimatic variability over the past two millennia »;

- Myriam Khodri (LOCEAN IRD/IPSL Sorbonne University, Paris) : « Modelling and understanding the influence of external forcings on climate variability and change over the past two millennia »;

- Johann Jungclaus (Max Planck Institute for Meteorology, Hamburg) : «The role of the ocean circulation in shaping European climate variations over the last millennium ».

\section{COURS À L'EXTÉRIEUR}

- Peter Wall Institute for Advanced Studies et Université de la ColombieBritannique, Vancouver, Canada.

- 7 septembre 2017 : «A paleoclimatic perspective on ocean oxygen minima »; - 12 septembre 2017 : "Climate evolution, sea-level rise and ice-sheet retreat during the last deglaciation »;

- 14 septembre 2017: "Radiocarbon as a geochronometer and as a tracer in paleoclimatology, geophysics and astrophysics ».

- Institut Max-Planck d'Anthropologie évolutionniste, Leipzig, Allemagne.

- 16 janvier 2018: «Abrupt sea level changes and consequences for the peopling of the americas »;

- 17 janvier 2018: «Marine reservoir age of radiocarbon: a problem and a proxy for paleoenvironmental changes ».

- Université de Mayence et Institut Max-Planck de Chimie, Allemagne.

- 24 mai 2018 : «Radiocarbon as a tool to probe the past oceans ».

\section{RECHERCHE}

Une partie du travail a porté sur les forçages climatiques au cours du dernier millénaire, notamment la variabilité solaire et l'influence des éruptions volcaniques. Le dernier millénaire préindustriel fait partie des périodes sélectionnées pour le projet de comparaison des modèles climatiques (sixième phase du projet de comparaison de modèles couplés CMIP et quatrième phase de PMIP). Les simulations transitoires des 1000 dernières années servent à étudier la réponse au forçage naturel dans des conditions de fond similaires à celles d'aujourd'hui, ainsi qu'à distinguer les variabilités forcées et internes du système. L'activité solaire au cours des derniers millénaires est reconstituée à partir des radionucléides cosmogéniques mesurés dans plusieurs archives terrestres (glaces polaires, bois des arbres). Nous avons comparé les séries du ${ }^{10} \mathrm{Be}$ obtenues pour l'Antarctique et le Groenland avec la reconstitution de la production globale fondée sur $1 \mathrm{e}{ }^{14} \mathrm{C}$ (Wu et al., 2018). Il est possible de distinguer deux états d'activité solaire : la composante principale, correspondant au 
niveau modéré «normal », et une composante secondaire correspondant aux grands minima d'activité.

Une partie importante de notre travail concerne l'influence des éruptions volcaniques sur l'indicateur ${ }^{10} \mathrm{Be}$ utilisé pour reconstituer l'activité solaire (Baroni et $a l$., soumis). Les mesures obtenues dans les puits de neige antarctiques indiquent une augmentation de la concentration en ${ }^{10} \mathrm{Be}$ datant des éruptions volcaniques stratosphériques d'Agung en 1963 et du Pinatubo en 1991. La quantité massive d'aérosols volcaniques formés dans la stratosphère a favorisé le drainage et le dépôt des atomes de ${ }^{10} \mathrm{Be}$ depuis le réservoir stratosphérique.

Nous avons étudié une carotte de glace couvrant le dernier millénaire ainsi que différents enregistrements provenant de sites en Antarctique. Une augmentation significative de la concentration en ${ }^{10} \mathrm{Be}$ au moment des éruptions stratosphériques est observée pour la majorité des événements volcaniques étudiés (Baroni et al., soumis). Les paramètres de régression linéaire entre les concentrations en ${ }^{10} \mathrm{Be}$ cosmogénique et en sulfate volcanique fournissent des informations importantes et complémentaires. Pour chaque éruption, la pente de la régression est un indicateur de l'efficacité du drainage des atomes de ${ }^{10} \mathrm{Be}$ par les aérosols volcaniques. L'éruption du Samalas en 1257 AD se distingue comme la plus grande éruption du dernier millénaire avec une pente faible. La persistance des aérosols volcaniques dans la stratosphère après l'éruption de Samalas a vraisemblablement drainé le réservoir stratosphérique de ${ }^{10} \mathrm{Be}$ pendant au moins une décennie.

En parallèle à ces travaux sur les forçages climatiques, nous avons étudié la réponse du climat au cours des derniers millénaires en analysant des sédiments lacustres. De nouveaux enregistrements paléoclimatiques ont été obtenus pour des sites dans les Alpes françaises, au Maroc et en Iran. L'approche illustre le potentiel de ces archives sédimentaires sensibles aux conditions hydroclimatiques pour évaluer l'impact des variations climatiques de la zone méditerranéenne et subtropicale.

L'étude d'une carotte sédimentaire prélevée dans le lac d'Allos (2 $200 \mathrm{~m}$ d'altitude) a été réalisée sur la base d'analyses sédimentologiques, géochimiques et micropaléontologiques (Cartier et al., 2018). Ces enregistrements ont permis d'identifier des changements majeurs qui se sont produits au cours de l'histoire paléoenvironnementale de ce bassin. Durant la période de l'Holocène, deux transitions ont été enregistrées vers 8600 et 3000 cal. BP par tous les compartiments écologiques, tandis que la période 4500-3000 cal. BP se distingue par des changements qui ne concernent que l'écosystème lacustre.

Les dépôts sédimentaires du lac Azigza (Moyen Atlas marocain, $1550 \mathrm{~m}$ d'altitude) ont été datés et analysés en combinant des mesures géochimiques et minéralogiques avec une caractérisation des microfaciès (Jouve et al., 2018). La composante détritique analysée par scanner XRF et étude des microstructures sédimentaires a fourni des indicateurs du ruissellement sur le bassin versant et des variations du niveau du lac. Ces indicateurs ont été calibrés avec les mesures hydroclimatiques instrumentales disponibles pour les cinquante dernières années. Il a ensuite été possible de reconstituer les changements hydrologiques depuis 1879.

Les analyses sédimentologiques et géochimiques d'une carotte prélevée dans le lac Maharlou (sud-ouest de l'Iran) ont été utilisées pour reconstituer les changements hydrologiques au cours des derniers millénaires (Brisset et al., 2018). Les sédiments de ce lac salé sont formés de lamines alternées constituées d'évaporites, de carbonates et de minéraux détritiques. Les données ont été interprétées en fonction 
de trois conditions hydrologiques principales : dessiccation du lac marquée par des couches de halite, conditions hypersalines marquées par une abondance de gypse, conditions humides caractérisées par des apports terrigènes. Des couches correspondant à des crues distinctes indiquent une activité fluviatile importante. Les variations hydrologiques du lac Maharlou à la fin de l'Holocène correspondent bien aux enregistrements de formation des dunes et des phases d'alluvionnement des rivières en Iran et dans la péninsule arabique, ainsi qu'aux enregistrements régionaux mesurés dans les spéléothèmes. En plus des influences climatiques sur l'hydrologie du lac, les sociétés humaines ont modifié le drainage de l'eau dans le bassin versant. Les périodes d'utilisation des tunnels souterrains (qanats) correspondent à des diminutions du volume d'eau du lac. Les changements climatiques ont entraîné une pénurie d'eau renforcée par les activités anthropiques, conduisant à une dessiccation plus fréquente du lac au cours du dernier millénaire.

\section{PUBLICATIONS}

BARd É., «Climate Tales », in P. Tortell, M. Turin et M. Young (dir.), Memory, Vancouver, UBC Press, Peter Wall Institute for Advanced Studies, 2018, p. 31-37.

Braucher R., Keddadouche K., Aumaître G., Bourlès D.L., Arnold M., Pivot S., BARONI M., SCHARF A., RUGEL G. et BARD É., « Chlorine measurements at the 5MV French AMS national facility ASTER: Associated external uncertainties and comparability with the 6MV DREAMS facility », Nuclear Instruments and Methods in Physics Research Section B: Beam Interactions with Materials and Atoms, vol. 420, 2018, p. 40-45, DOI : 10.1016/j. nimb.2018.01.025.

Brisset E., Djamali M., Bard É., Borschneck D., Gandouin E., Garcia M., Stevens L. et TACHIKAWA K., «Late Holocene hydrology of Lake Maharlou, southwest Iran, inferred from high-resolution sedimentological and geochemical analyses », Journal of Paleolimnology, 2018, DOI : 10.1007/s10933-018-0048-6.

Capano M., Miramont C., Guibal F., Kromer B., Tuna T., Fagault Y. et Bard É., «Wood 14C dating with AixMICADAS: Methods and application to tree-ring sequences from the Younger Dryas event in the southern French Alps », Radiocarbon, vol. 60, $\mathrm{n}^{\circ}$ 1, 2018, p. 51-74, DOI : 10.1017/RDC.2017.83.

Cartier R., Brisset E., Guiter F., Sylvestre F., Tachikawa K., Anthony E.J., Pailles C., Bruneton H., Bard É. et Miramont C., « Multiproxy analyses of Lake Allos reveal synchronicity and divergence in geosystem dynamics during the Lateglacial/Holocene in the Alps ", Quaternary Science Reviews, vol. 186, 2018, p. 60-77, DOI : 10.1016/j. quascirev.2018.02.016.

DAVTIAN N., BARD É., MÉNOT G. et FAgAult Y., «The importance of mass accuracy in selected ion monitoring analysis of branched and isoprenoid tetraethers », Organic Geochemistry, vol. 118, 2018, p. 58-62, DOI : 10.1016/j.orggeochem.2018.01.007.

Fewlass H., Talamo S., Tuna T., Fagault Y., Kromer B., Hoffmann H., Pangrazzi C., HUBLIN J.-J. et BARD É., «Size matters : radiocarbon dates of $<200 \mu \mathrm{g}$ ancient collagen samples with AixMICADAS and its gas ion source », Radiocarbon, vol. 60, n 2, 2018, p. 425 439, DOI : 10.1017/RDC.2017.98.

Jouve G., Vidal L., Adallal R., Rhoujuati A., Benkaddour A., Chapron E., Tachikawa K., Bard É., Courp T., Dezileau L., Hebert B., RapuC W., Simonneau A., SONZOGNI C. et SYLVESTRE F., «Recent hydrological variability of the Moroccan Middle Atlas Mountains inferred from microscale sedimentological and geochemical analyses of lake sediments », Quaternary Research, 2018, p. 1-17, DOI : 10.1017/qua.2018.94. 
Tuna T., Fagault Y., Bonvalot L., Capano M. et Bard É., « Development of small $\mathrm{CO}_{2}$ gas measurements with AixMICADAS », Nuclear Instruments and Methods in Physics Research Section B: Beam Interactions with Materials and Atoms, vol. 437, 2018, p. 93-97, DOI : 10.1016/j.nimb.2018.09.012.

Wu C.J., Usoskin I.G., Krivova N., Kovaltsov G.A., BARONi M., BARD É. et SOlAnKi S.K., « Solar activity over nine millennia: A consistent multi-proxy reconstruction », Astronomy \& Astrophysics, vol. 615, 2018, A93, DOI : 10.1051/0004-6361/201731892. 
